\title{
Deuterium content and site occupancy in iron sulphide at high pressure and high temperature: Implications for the oxidation of early Earth's mantle
}

\author{
SUMITH ABEYKOON ${ }^{1}$, CHRISTOPHER HOWARD ${ }^{1}$, \\ SERENA DOMINIJANNI ${ }^{1}$, LISA EBERHARD ${ }^{1}$, DANIEL \\ FROST $^{1}$, TIZIANA BOFFA BALLARAN ${ }^{1}$, ALEXANDER \\ KURNOSOV $^{1}$, HIDENORI TERASAKI ${ }^{2}$, TATSUYA \\ SAKAMAKI $^{3}$, AKIO SUZUKI ${ }^{3}$, EIJI OHTANI ${ }^{3}$, ASAMI \\ SANO-FURUKAWA ${ }^{4}$ AND JUN ABE ${ }^{5}$ \\ ${ }^{1}$ University of Bayreuth \\ ${ }^{2}$ Okayama University \\ ${ }^{3}$ Tohoku University \\ ${ }^{4}$ J-PARC Center, Japan Atomic Energy Agency \\ ${ }^{5}$ Neutron Science and Technology Center, CROSS \\ Presenting Author: sumith.abeykoon@uni-bayreuth.de
}

The rapid increase in the oxidation state of the Earth's mantle after core formation [1] was a key process in Earth's development towards habitability. One possibility is that the mantle was oxidised by water, through the reaction $\mathrm{H}_{2} \mathrm{O}+2 \mathrm{FeO}=$ $\mathrm{Fe}_{2} \mathrm{O}_{3}+\mathrm{H}_{2}$, delivered during the late stages of accretion $[2,3]$. However, the resulting $\mathrm{H}_{2}$ needs to be removed from the mantle to prevent it from reversing the oxidation process. The main phase segregating to the core during the final period of accretion is proposed to be iron sulphide [4]. A viable mechanism to remove $\mathrm{H}_{2}$ from the mantle would be for it to be incorporated into this $\mathrm{FeS}$ melt. Investigating the hydrogen incorporation into $\mathrm{FeS}$ is only possible via in-situ methods at high-pressures $(P)$ and high-temperatures $(T)$, since $\mathrm{H}_{2}$ exsolves from $\mathrm{FeS}$ during quenching.

We performed in-situ high-pressure time-of-flight neutron diffraction experiments on the FeS-H(D) system using a six-axis multi-anvil press installed at the PLANET beamline (BL-11), $J$ $P A R C$, Japan. Thermal decomposition of deuterated ammonia borane $\left(\mathrm{ND}_{3} \mathrm{BD}_{3}\right)$ supplied deuterium to the FeS starting material upon heating at high- $P$. Powder diffraction profiles were acquired over $P$ and $T$ ranges of 3-12 GPa and 700-1200 K. Site occupancies of deuterium in the FeS structure were determined with Rietveld structure refinement.

The NiAs-type FeS V structure [5] was used to test different models. Deuterium was found to substitute on to two interstitial sites and the total occupancy of deuterium was up to $\sim 0.77 \pm 0.12$ atoms per FeS formula unit at $6.9 \mathrm{GPa}$ and $960 \mathrm{~K}$. Furthermore, deuteration of FeS observed over time is consistent with unit-cell volume evolution at the above conditions. Given that the $\mathrm{H}$ content of FeS liquid is likely higher than in solid FeS, our current results suggest that $\mathrm{FeS}$ is a viable candidate for segregating hydrogen to the core during the late stage of core formation, which would facilitate mantle oxidation by $\mathrm{H}_{2} \mathrm{O}$.

[1] Kasting et al. (1993), J. Geol. 101, 245-257

[2] Ringwood (1977), Geochem. J. 11, 111-135

[3] Sharp et al. (2013) EPSL 380, 88-97.

[4] O’Neill (1992) GCA 55, 1159-1172 